\title{
Original
}

\section{Estimation of the disposition index based on components of metabolic syndrome}

\author{
Jiunn-Diann Lin ${ }^{1)}$, Chang Hsun Hseih ${ }^{2)}$, Chuan Chieh Liu ${ }^{3)}$, Wei-Cheng Lian ${ }^{4)}$, Chung-Ze Wu ${ }^{1)}$, \\ Chun-Hsien $\mathrm{Hsu}^{5)}$, Dee Pei ${ }^{6}$, Te-Lin $\mathrm{Hsia}^{6)}$ and Yen-Lin Chen ${ }^{7)}$ \\ 1) Division of Endocrinology, Department of Internal Medicine, Shuang Ho Hospital, School of Medicine, Taipei Medical University, \\ Taipei, $R O C$ \\ 2) Division of Endocrinology and Metabolism, Department of Internal Medicine, Tri-Service General Hospital, Taipei, ROC \\ 3) Department of Internal Medicine, Cardinal Tien Hospital, School of Medicine, Fu-Jen Catholic University, New Taipei City, ROC \\ 4) Division of Endocrinology and Metabolism, Department of Internal Medicine, Buddhist Dalin Tzu Chi General hospital, School of \\ Medicine, Taiwan, ROC \\ 5) Department of Family Medicine, Cardinal Tien Hospital, School of Medicine, Fu-Jen Catholic University, New Taipei City, ROC \\ 6) Division of Endocrinology and Metabolism, Department of Internal Medicine, Cardinal Tien Hospital, School of Medicine, \\ Catholic Fu Jen University, New Taipei City, ROC \\ 7) Department of Pathology, Cardinal Tien Hospital, School of Medicine, Fu Jen Catholic University, New Taipei City, ROC
}

\begin{abstract}
Decreased insulin sensitivity (IS) and impaired insulin secretion are major pathological features of type 2 diabetes (T2DM). The product of these factors is the disposition index (DI). We aimed to develop an equation for predicting DI. We enrolled 167 participants in our study. We randomly assigned $126(75 \%)$ of the participants to the study group, whose data would be used to build the equation for estimating the DI. The remaining 41 participants comprised the external validation group. A frequently sampled intravenous glucose-tolerance test was performed for all participants, and the IS, the glucose sensitivity, the acute insulin response to the glucose load, and the DI were determined. Three factors were selected from multiple linear regression analysis, and we constructed the equation $\log (\mathrm{DI})=2.449-0.113 \times$ fasting plasma glucose + $0.046 \times$ body mass index $-0.612 \times$ high-density lipoprotein cholesterol. Using this equation, the calculated log (DI) significantly correlated with the measured $\log (\mathrm{DI})$ in the external validation group $(\mathrm{r}=0.428, p=0.007)$. By using the equation based on the demographic data and measurements of metabolic syndrome components, the DI could be predicted with acceptable accuracy $(\mathrm{r}=0.428)$. Because of the relationships between the MetS and demographic parameters, this method of predicting DI may help further clinicians' understanding of the underlying pathological mechanisms in T2DM.
\end{abstract}

Key words: Disposition index, Metabolic syndrome, Insulin sensitivity

TYPE 2 DIABETES MELLITUS (T2DM) is 1 of the 5 most common causes of death in Taiwan and many other developed countries, and the morbidity and mortality from T2DM are a tremendous health care burden. Thus, identifying patients at high risk for T2DM has become an important task for health care providers. It is estimated that approximately $10 \%$ of people over 60 years of age are unaware that they have undiagnosed T2DM [1]. Because of metabolic memory [2], this group of people is at risk of losing the opportunity for early intervention, which would likely improve their prognosis.

Submitted Nov. 30, 2013; Accepted May 8, 2014 as EJ13-0510 Released online in J-STAGE as advance publication May 28, 2014 Correspondence to: Yen-Lin Chen, Te-Lin Hsia, Cardinal Tien Hospital, No 362, Chung Cheng Road, Xindian, Taipei County 23137, Taiwan, R.O.C. E-mail: anthonypatho@gmail.com Abbreviations: T2DM, type 2 diabetes mellitus; IS, insulin sensitivity;
Impaired insulin secretion and decreased insulin sensitivity (IS), which are conceptually the reciprocal of insulin resistance (IR), are the 2 major pathological features of T2DM [3-4]. The product of these 2 factors, IS and acute insulin response after glucose load (AIRg), is the disposition index (DI). From a pathophysiological viewpoint, patients must have impairment of both factors to be diagnosed with T2DM [4]. At the same time, DI is a well-known predictor of T2DM [5]. The IS and AIRg are often determined using the frequently sampled intravenous glucose-tolerance test (FSIGT).

IR, insulin resistance; DI, disposition index; AIRg, acute insulin response after glucose load; FSIGT, frequently sampled intravenous glucose-tolerance test; MetS, metabolic syndrome; FPG, fasting plasma glucose; BMI, body mass index ; NGT, normal glucose tolerance; pre-T2DM, pre-diabetes; TG, triglyceride; HDL-C, highdensity lipoprotein cholesterol; FPI, fasting plasma insulin. 
However, although accurate, the FSIGT is labor-intensive and time-consuming, and clinicians at many health care facilities do not have the ability to perform the FSIGT. Consequently, the diagnostic importance of the DI is often not emphasized in clinical practice.

Previous studies have shown that IR is the core feature of metabolic syndrome (MetS) [6-9]. In the same time, insulin secretion is also tightly related to age and individual MetS components in non-diabetic subjects $[6,10]$. More importantly, these components are routinely assessed in many hospitals and research facilities. Thus, we hypothesized that the DI could be accurately predicted by using these components in people with or without MetS. We believed that the prediction of DI may further help clinicians' to understand the underlying pathophysiologies of T2DM.

\section{Methods}

\section{Participants}

We enrolled 167 participants in our study and all of them received both FSIGT and $75 \mathrm{~g}$ standard oral glucose tolerance test (OGTT). Participants were either self-referred or referred by health professionals with whom they had requested screening for T2DM. They had no history of T2DM, and had not previously used medications for T2DM. The participants were stratified as the groups with normal glucose tolerance (NGT), pre-diabetes (pre-T2DM), or T2DM according to the criteria established by the American Diabetes Association in 2012 [11]. None of the participants had a significant medical or surgical history. Before participation in our study, the participants were instructed by physicians and dietitians to refrain from using any medication known to affect glucose or lipid metabolism, and to stay on a stable diet for at least 1 week before the start date of the study. On Day 1 of the study, participants underwent a complete routine work-up to exclude patients with cardiovascular, endocrine, renal, hepatic, or respiratory disorders. Our study protocol was approved by the Institutional Review Board and the Ethics Committee at Cardinal Tien Hospital (CTH-101-2-5-028). All participants provided written informed consent prior to participation. From the 167 participants, $126(75 \%)$ were randomly assigned to the study group. Based on these participants' characteristics, an equation was constructed for estimating the DI. This equation was used to calculate the DI in the remaining 41 participants as an external validation.

\section{Study protocol}

All tests were performed in the Clinical Research Center of our institution. On the day of the OGTT, after a 10-hour overnight fast, a standard $75 \mathrm{~g}$ OGTT was performed at 8:00 AM. Blood was drawn before the glucose load and at and 120 minutes after the glucose load for the measurements of plasma glucose. At least one week apart from the first study visit, FSIGT was performed. After a 12-hour overnight fasting period, one intravenous (IV) catheter was placed in each arm of the participants, through which a bolus of a $10 \%$ glucose solution $(0.3 \mathrm{~g} / \mathrm{kg})$ was administered. A bolus of regular human insulin (Novo Nordisk Pharmaceutical, Princeton, NJ, USA) was also administered IV at 20 minutes after the glucose load. Blood samples for plasma glucose and insulin testing were collected at 0 , $2,4,8,19,22,30,40,50,70,100$, and 180 minutes. The IS, glucose sensitivity, and the AIRg were calculated using the Bergman minimal model [4], with the DI as the product of the IS and the AIRg.

Plasma was separated within 1 hour of blood sample collection, and stored at $-30^{\circ} \mathrm{C}$ until analysis. The plasma glucose was measured using a YSI Model 203 glucose analyzer based on the oxidase method (Yellow Spring Instrument, Yellow Spring, Ohio, USA). The plasma insulin level was determined using the Coat-ACount solid phase radioimmunoassay kit (Diagnostic Products, Los Angeles, CA, USA), with intra- and inter-assay coefficients of variance of $3.3 \%$ and $2.5 \%$, respectively. The serum triglyceride (TG) was measured using the dry multilayer analytical slide method in a Fuji Dri-Chem 3000 analyzer (Fujifilm, Tokyo, Japan). Serum high-density lipoprotein cholesterol (HDL-C) was determined using an enzymatic cholesterol assay following dextran sulfate precipitation.

\section{Statistical analysis}

The data were tested for normal distribution using the Kolmogorov-Smirnov test, and homogeneity of the variances was assessed using the Levene test. The continuous variables were expressed as mean \pm standard deviation. The DI, the AIRg, the IS, and the fasting plasma insulin (FPI) were not normally distributed, and were logarithmically transformed. An independent $t$ test was used to evaluate the demographic data, clinical characteristics, and parameters derived from the FSIGT between the 2 groups. One-way analysis of variance was used to compare the differences in the clinical parameters among the participants with NGT, 
Table 1 Demographic data of the study group and the external validation group

\begin{tabular}{|c|c|c|c|}
\hline & Study Group & ExtV Group & $p$ value \\
\hline $\mathrm{n}$ & 126 & 41 & \\
\hline Sex (Male/Female) & $62 / 64$ & $19 / 22$ & 0.858 \\
\hline Age $(y)$ & $49.8 \pm 14.5$ & $52.7 \pm 10.7$ & 0.175 \\
\hline Body mass index $\left(\mathrm{kg} / \mathrm{m}^{2}\right)$ & $25.3 \pm 4.5$ & $25.5 \pm 3.4$ & 0.830 \\
\hline Systolic blood pressure $(\mathrm{mmHg})$ & $120.9 \pm 13.9$ & $121.3 \pm 13.9$ & 0.879 \\
\hline Diastolic blood pressure (mmHg) & $76.0 \pm 7.5$ & $74.5 \pm 9.2$ & 0.313 \\
\hline Triglyceride $(\mathrm{mmol} / \mathrm{L})$ & $1.3 \pm 0.6$ & $1.2 \pm 0.5$ & 0.410 \\
\hline $\mathrm{HDL}-\mathrm{C}(\mathrm{mmol} / \mathrm{L})$ & $1.1 \pm 0.3$ & $1.1 \pm 0.4$ & 0.493 \\
\hline Fasting plasma glucose $(\mathrm{mmol} / \mathrm{L})$ & $7.7 \pm 2.9$ & $7.8 \pm 2.8$ & 0.802 \\
\hline Log of fasting plasma insulin (pmol/L) & $1.3 \pm 0.6$ & $1.4 \pm 0.5$ & 0.622 \\
\hline Log of acute insulin response after glucose load $(\mu \mathrm{U} / \mathrm{min})$ & $2.0 \pm 0.9$ & $1.9 \pm 0.9$ & 0.464 \\
\hline Log of insulin sensitivity $\left(10^{-4} \cdot \mathrm{min}^{-1} \cdot \mathrm{pmol}^{-1} \cdot \mathrm{L}^{-1}\right)$ & $0.1 \pm 0.6$ & $0.2 \pm 0.6$ & 0.258 \\
\hline Log of Disposition index & $2.1 \pm 0.9$ & $2.1 \pm 0.9$ & 0.986 \\
\hline Glucose sensitivity $\left(10^{-2} \cdot \mathrm{dL} \cdot \mathrm{min}^{-1} \cdot \mathrm{kg}^{-1}\right)$ & $0.016 \pm 0.010$ & $0.014 \pm 0.009$ & 0.233 \\
\hline
\end{tabular}

Data are expressed as mean \pm standard deviation. Ext V group, external validation group; HDL-C, high-density lipoprotein cholesterol

Table 2 Demographic data of normal glucose tolerance, pre-diabetes, and diabetes participants

\begin{tabular}{|c|c|c|c|}
\hline & Normal glucose tolerance & Pre-diabetes & diabetes \\
\hline $\mathrm{n}$ & 46 & 38 & 83 \\
\hline Age (y) & $42.4 \pm 17.4^{2,3}$ & $54.4 \pm 11.8^{1}$ & $53.2 \pm 10.1^{1}$ \\
\hline Body mass index $\left(\mathrm{kg} / \mathrm{m}^{2}\right)$ & $26.3 \pm 6.0$ & $24.9 \pm 3.1$ & $25.1 \pm 3.4$ \\
\hline Systolic blood pressure (mmHg) & $118.0 \pm 11.1$ & $120.9 \pm 14.6$ & $122.5 \pm 14.7$ \\
\hline Diastolic blood pressure $(\mathrm{mmHg})$ & $74.1 \pm 7.0$ & $75.7 \pm 8.4$ & $76.5 \pm 8.2$ \\
\hline Triglyceride $(\mathrm{mmol} / \mathrm{L})$ & $1.2 \pm 0.6$ & $1.4 \pm 0.6$ & $1.3 \pm 0.5$ \\
\hline HDL-C (mmol/L) & $1.1 \pm 0.4$ & $1.2 \pm 0.3$ & $1.1 \pm 0.3$ \\
\hline Fasting plasma glucose $(\mathrm{mmol} / \mathrm{L})$ & $4.7 \pm 0.4^{2,3}$ & $6.4 \pm 0.4^{1,3}$ & $10.0 \pm 2.3^{1,2}$ \\
\hline Log of fasting plasma insulin $(\mathrm{pmol} / \mathrm{L})$ & $1.5 \pm 0.7$ & $1.3 \pm 0.7$ & $1.3 \pm 0.6$ \\
\hline Log of acute insulin response after glucose load $(\mu \mathrm{U} / \mathrm{min})$ & $2.8 \pm 0.8^{2,3}$ & $1.9 \pm 0.7^{1}$ & $1.6 \pm 0.8^{1}$ \\
\hline $\log$ of insulin sensitivity $\left(10^{-4} \cdot \mathrm{min}^{-1} \cdot \mathrm{pmol}^{-1} \cdot 1^{-1}\right)$ & $0.11 \pm 0.51$ & $0.15 \pm 0.62$ & $0.10 \pm 0.49$ \\
\hline Log of disposition index & $2.8 \pm 0.7^{2,3}$ & $2.0 \pm 0.9^{1}$ & $1.7 \pm 0.8^{1}$ \\
\hline Glucose sensitivity $\left(10^{-2} \cdot \mathrm{dL} \cdot \mathrm{min}^{-1} \cdot \mathrm{kg}^{-1}\right)$ & $0.020 \pm 0.010^{2,3}$ & $0.014 \pm 0.008^{1}$ & $0.014 \pm 0.010^{1}$ \\
\hline
\end{tabular}

pre-T2DM, and T2DM. The Bonferroni test was used for post hoc examination.

The correlations between the MetS components and the DI were evaluated in the study group first and only factors which were significantly related to DI were selected to build the DI equation by using the stepwise multiple linear regression analysis. The data of clinicalmetabolic variables, including either the MetS component measurements or age and sex for the study-group participants were treated as independent variables, and the measured DI was treated as a dependent variable. The equation derived was used to calculate the DI values for the participants in the external validation group. The correlation between the calculated DI and the measured DI was evaluated using the Pearson correlation analysis, for which a higher value for the correlation coefficient (r) indicates a more accurate prediction. All statistical analyses were performed using the SPSS software package, version 13.0 (IBM, Armonk, NY, USA). A $p$ value less than .05 was considered to indicate a statistically significant relationship.

\section{Results}

The study group and the external validation group contained 126 and 41 participants, respectively. The demographic data, the fasting plasma glucose (FPG) with insulin (FPI), the plasma lipid levels, and the variables derived from the FSIGT for the study and the external validation groups are presented in Table 1. No significant differences were observed among these variables between the study and external validation groups. The demographic data for participants with NGT, pre-T2DM, and T2DM are shown in Table 2. The participants with 
Table 3 Pearson correlation analysis of the clinical parameters and the disposition index in the study group

\begin{tabular}{lrc}
\hline Variables & $\mathrm{r}$ & $p$ value \\
\hline Age & -0.325 & 0.000 \\
Body mass index & 0.217 & 0.019 \\
Systolic blood pressure & -0.032 & 0.739 \\
Diastolic blood pressure & 0.030 & 0.755 \\
Triglyceride & -0.085 & 0.369 \\
HDL-C & -0.208 & 0.032 \\
Fasting plasma glucose & -0.435 & 0.000 \\
Log (Fasting plasma insulin) & 0.149 & 0.108 \\
Log (Acute insulin response after glucose load) & 0.804 & 0.000 \\
Log (Insulin sensitivity) & 0.383 & 0.015 \\
\hline
\end{tabular}

HDL-C, high-density lipoprotein cholesterol

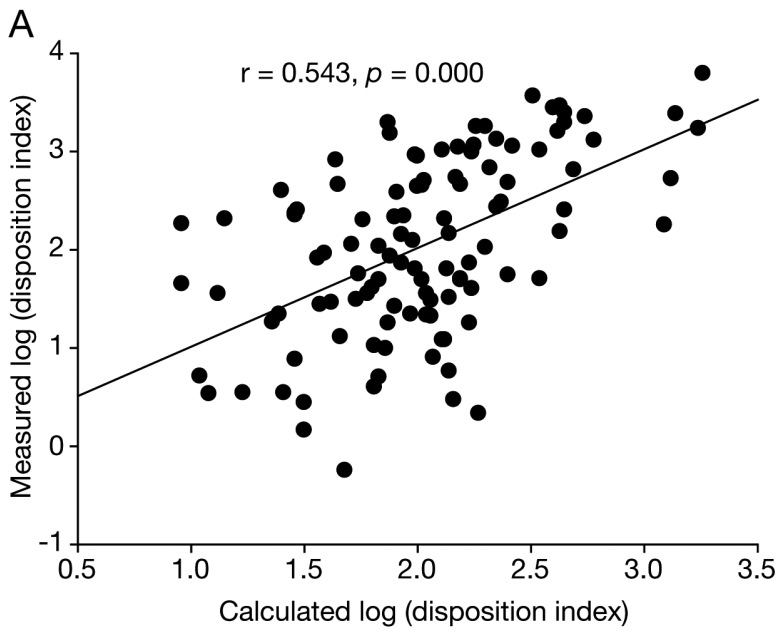

Fig. 1 The correlation between the calculated disposition index and the measured disposition index in the study group (Panel A) and external validation group (Panel B).

T2DM had lower AIRg and DI than those with NGT.

The correlations between the different parameters and the DI were evaluated in the study group to determine which contribute most to the DI, and the results are shown in Table 3. There were significant correlations between the DI and age $(\mathrm{r}=-0.325, p=0.000)$, body mass index (BMI) ( $\mathrm{r}=0.217, p=0.019)$, HDL-C $(\mathrm{r}=-0.208, p$ $=0.032)$, and FPG $(\mathrm{r}=-0.435, p=0.000)$. However, significant correlations between the DI and systolic blood pressure, diastolic blood pressure, triglycerides, or FPI were not found. It should be noted that, in both the study and external validation group, FPG was the best correlated with measured DI among these clinical-metabolic variables $(\beta=-0.391, p=0.000)$ (Table 4$)$. Only parameters that were significantly correlated were included in the multiple linear regression analysis, from which the equation ' $\log (\mathrm{DI})=2.449-0.113 \times \mathrm{FPG}+$

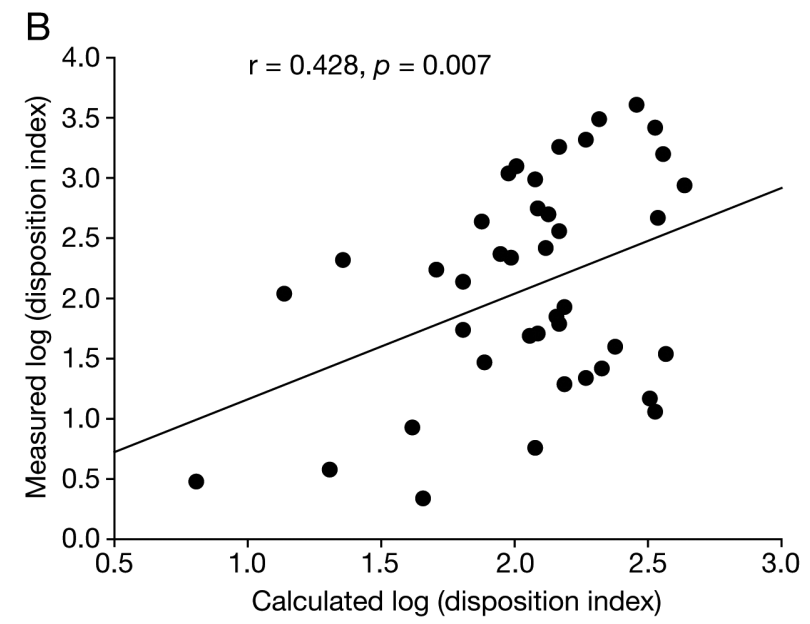

Table 4 Multiple linear regression of the factors associated with the logarithmically transformed disposition index of the study group

\begin{tabular}{lcc}
\hline Variables & $\beta$ & $p$ value \\
\hline Fasting plasma glucose & -0.391 & 0.000 \\
Body mass index & 0.244 & 0.006 \\
HDL-C & -0.198 & 0.024 \\
\hline
\end{tabular}

Beta, standardized coefficients; HDL-C, high-density lipoprotein cholesterol

$0.046 \times \mathrm{BMI}-0.612 \times \mathrm{HDL}-\mathrm{C}^{\prime}$ was constructed. Based on this equation, the DI of the external validation group was calculated. The correlation between the calculated DI and the measured DI was assessed by linear regression. Since they are not normally distributed, log transformation was performed and they are significantly correlated both in the study group (Fig. 1, Panel A) and in the external validation group (Fig. 1, Panel B).

\section{Discussion}

This is the first study to build an algebraic equation and evaluate its accuracy in predicting the DI in subjects with various degrees of glucose tolerance. Although previous studies have predicted IS or AIRg, most enrolled participants without diabetes only [6, 12-15]. Although our method of predicting DI did not 
demonstrate a high level of accuracy $(r=0.428$ in the external validation group), we nonetheless believe that this equation can be used in patients over the reference range of glucose tolerance, enabling the early identification of susceptibility to T2DM. However, one still may argue that in the simple correlation, it could be noted that FPG was the most important factor to predict DI, then why bother to use a more complicated equation to estimate DI? In fact, it is important to note that FPG is driven by two forces; i.e., IR and insulin secretion. Therefore, conceptually, FPG is less powerful evaluator for glucose metabolism than DI. Our data also proved this hypothesis. By using the hierarchical multiple regression method, we have shown that DI has a better predictability power than FPG alone. Thus, we believe that our data aid in the understanding of the role of the DI in the progression to T2DM.

Previous studies have predicted IS based on demographic characteristics and routine biochemical data $[6$, 12-14, 16-18]. However, the clamp method remains the gold standard, and is generally considered to provide more comprehensive information. Using the clamp technique, van Haeften et al. demonstrated that IS could be estimated based on clinical-metabolic variables $\left(r^{2}=0.160\right.$ in the subjects with NGT and $r^{2}=$ 0.449 in the subjects with impaired glucose-tolerance, respectively) [19]. In addition, Chiu et al. reported that a multiple linear regression equation demonstrated that various demographic data (diastolic blood pressure, ethnicity, sex, age, and waist-hip ratio) were correlated with IS measured using the same method $\left(\mathrm{r}^{2}\right.$ $=0.4366)$ in participants with NGT [14]. In these 2 studies, easily obtainable demographic data were used. Because hyperinsulinemia is positively correlated with IR, increases in the values for plasma insulin level raise the predictive accuracy of the multiple regression equation. Other commonly used methods, such as the HOMA-IR and QUICKI indices, include the plasma insulin level $[16,20]$. However, measuring the plasma insulin level is an expensive procedure, and is not a routine biochemical analysis. Thus, the use of the plasma insulin level as a diagnostic tool has not been practical for most clinicians, and has been primarily limited to research.

The AIRg is generally considered to represent the first phase of insulin secretion (1stISEC) because it indicates the level of insulin stored in the pancreas granules. The most widely used methods for measuring 1stISEC are the hyperglycemic clamp test and the
FSIGT. Previous studies have shown that sex, age, family history (FH), waist-hip ratio, BMI, and FPG are predictors of 1stISEC [6, 14-15, 18]. However, among these studies, the strongest correlation was reported by Alford et al., who used FH and FPG in their analysis. Similar to the estimation of IS, there are also surrogates that include the plasma insulin level as a factor in highly accurate measurements of 1stISEC, such as the Stumvoll and insulinogenic indices [17, 21-22]. However, the use of these methods has also been limited because of the need for measuring the plasma insulin level.

As aforementioned, hyperglycemia is associated with deterioration of both IS and $\beta$-cell function. Hanley et al. reported that the FPG was negatively correlated with both the IS $(r=-0.33)$ and the AIRg $(r=$ -0.26) in participants without T2DM [6]. Alford et al. also reported a similar correlation between these factors $(r=-0.49$ and $r=-0.33$, respectively) in participants without T2DM [15]. These findings indicate that, among all other MetS components, FPG represents the statistically strongest predictor of the DI, which was consistent with our findings.

It has been well-established that increased BMI is associated with deterioration of IS but, in the same time, higher $\beta$-cell mass which is a synonym for better insulin secretion [23]. Hanley et al. observed that BMI had a greater effect on IS $(r=-0.54)$ than on AIRg $(r=0.14)$ and this led to its negative relationship with DI $(r=-0.35)$. Contrary to their study, our finding showed that $r$ value between BMI and AIRg was higher than that between BMI and IS $(r=0.377$, and $r=-0.254$, respectively) (See Supplemental Table I). This resulted in a positive correlation between BMI and DI $(\beta=0.244)$. This discrepancy could be explained by three possibilities. First, the degrees of obesity were originally different between Caucasians and Asians with T2DM [10,14]. In our study, the BMI was lower than that of Hanley et al. study (25.4, 28.0$30.0 \mathrm{~kg} / \mathrm{m}^{2}$, respectively). This would affect the relationships between BMI and DI. Second, the ability of $\beta$-cell compensation for IR might be different between Asians and Caucasians. This hypotheses is supported by the Torrens et al. study which demonstrated that the ability of $\beta$-cell function to compensate IR would vary in different ethnicities [24]. Finally, Hanley et al. only enrolled participants without diabetes. In the same time, subjects with various degrees of glucose tolerance were all included in our study. This could 
widen the range of DI and contributed to the differences between two studies.

In contrast, the effect of HDL-C on the values of AIRg and IS remains unclear. Theoretically, subjects with high HDL-C should have higher IS and AIRg [2526]. Reduced HDL-C is associated with impaired IS and with elevation of high plasma free fatty acid levels which is known to could cause the damage of $\beta$-cell [6]. At the same time, some other studies have demonstrated that HDL-C could promote insulin secretion and inhibit $\beta$-cell apoptosis [27]. In our study, a significant and negative correlation was found between HDL-C and DI (Table 4) which is opposite to the results of Hanley et al $(\mathrm{r}=0.16)$. To analyze our findings in details, it could be noted that this negative significance came from the correlation between HDL-C and AIRg $(\mathrm{r}=-0.198, p=$ $0.039)$ since the relationship between HDL-C and IS was non-significant $(\mathrm{r}=-0.077, p=0.424)$. The differences between these two studies could be explained by the following possibilities. First, it is well-known that the HDL-C level is affected by various phenotypes, including degree of obesity, IS and $\beta$-cell function [28-30]. This diversities could further be transferred down to intensity of the interactions between HDL-C and other clinical characteristics of diabetes such as IS and AIRg [10,14]. Secondly, as aforementioned, our enrollment of people with pre-T2DM and T2DM might also have contribution. It is interesting to note that the HDL-C level maintained the same as the FPG increased in our study (data are not shown). In the same time, unlike HDL-C level, the AIRg decreased significantly. This abrupt disappearing of the AIRg in patients with pre-T2DM and T2DM could explain the negative relationship between HDL-C and AIRg.

The FPI has been reported to be correlated with the IR, the ISEC, and the DI in participants without T2DM [6]. However, although we found significant associations between the IS and the FPI $(r=-0.378, p=$ $0.000)$ and between the AIRg and the FPI $(r=0.275, p$ $=0.002$ ) in our study (See Supplemental Table I), we found no significant correlation between the FPI and the DI. Ethnic-related differences between our cohort and those of previous studies may also have contributed to differences in these results, compared with those of previous studies. In addition, different inclusion criteria for participant in our study may have further contributed to the inconsistencies between our findings and those by Hanley et al. Moreover, plasma insulin levels may not be an optimal marker for IR or insulin secretion because "glucose toxicity" masks the true relationships between them in patients with T2DM with high blood glucose levels [31]. Half of our enrolled participants had T2DM, which may have caused the FPI to be a suboptimal predictor of the DI.

To our knowledge, no study has been conducted on the use of routine blood biochemical data to predict the DI directly. However, using clinically available information, such as demographic data and the measurement of MetS components, we showed that the IS and the AIRg could be predicted with the $r^{2}$ value of approximately 0.44 and 0.27 , respectively [14-15]. Despite an $\mathrm{r}^{2}$ for predicting the DI of 0.295 in the study group and 0.183 in the external validation group, our results are clinically relevant. The current consensus is that both impaired IS and insulin secretion are required for T2DM to develop. Therefore, the DI should be a better predictor for T2DM than either the IS or the AIRg.

One possible limitation of our findings was that the equation was based on the reference range of glucose tolerance. Because the relative contribution of the clinical-metabolic variables to the DI may differ with various degrees of glucose intolerance, the equation would differ among the groups with NGT, pre-T2DM, and T2DM. However, because our study cohort was relatively small, we were unable to stratify these participants into 3 groups. In addition, we did not include the FH of T2DM in our analysis, which is known to be an important risk factor for T2DM [13, 32]. However, the inclusion of $\mathrm{FH}$ in our analysis may improve the predictive accuracy of our equation. Furthermore, the results of our cross-sectional study may lack statistical power, compared with those of a longitudinal study. Further longitudinal studies with larger cohorts are needed to confirm our findings.

In conclusion, we constructed an equation based on demographic data and routine clinical measurements of MetS components that predicted the DI in patients with various degrees of glucose tolerance with an acceptable level of accuracy $(r=0.428)$. In addition, because of the relationships between the MetS and demographic parameters upon which the equation is based, its use for the prediction of DI may help further clinicians' understanding of the underlying pathological mechanisms in T2DM.

\section{Funding}

None. 


\section{Acknowledgements}

The authors thank all subjects who participated in the study.

\section{Declaration of Interest}

No conflict of interest.

\section{Authors Contributions}

Hsieh Chang-Hsun researched data. Lin JiunnDiann wrote the manuscript. Wu Chung-Ze and Chen Yen-Lin reviewed and edited the manuscript. Pei Dee contributed to the discussion and edited the manuscript. Wei-Cheng Lian, Liang Yao-Jen, Hsu ChunHsien, An-Tsz Hseih and Chuan Chieh Liu researched data and contributed to discussion.

Supplemental Table 1 Pearson Correlation between the clinical parameters and insulin sensitivity and acute insulin response after glucose load in the study group

\begin{tabular}{|c|c|c|}
\hline Variables & $\log (\mathrm{SI})(p$ value $)$ & Log AIRg ( $p$ value) \\
\hline Age & $0.162(0.074)$ & $-0.380(0.010)$ \\
\hline BMI & $-0.254(0.005)$ & $0.377(0.000)$ \\
\hline SBP & $-0.131(0.156)$ & $0.005(0.956)$ \\
\hline DBP & $-0.083(0.380)$ & $-0.007(0.941)$ \\
\hline TG & $-0.155(0.090)$ & $0.061(0.510)$ \\
\hline HDL-C & $-0.077(0.424)$ & $-0.198(0.039)$ \\
\hline FPG & $-0.020(0.828)$ & $-0.504(0.000)$ \\
\hline $\log (\mathrm{FPI})$ & $-0.378(0.000)$ & $0.275(0.002)$ \\
\hline $\log (A I R g)$ & $-0.198(0.026)$ & 1 \\
\hline $\log (\mathrm{IS})$ & 1 & $-0.198(0.026)$ \\
\hline
\end{tabular}

\section{References}

1. Harris MI, Flegal KM, Cowie CC, Eberhardt MS, Goldstein DE, et al. (1998) Prevalence of diabetes, impaired fasting glucose, and impaired glucose tolerance in U.S. adults. The Third National Health and Nutrition Examination Survey, 1988-1994. Diabetes Care 21: 518-524.

2. Ceriello A, Ihnat MA, Thorpe JE (2009) Clinical review 2: The "metabolic memory": is more than just tight glucose control necessary to prevent diabetic complications? J Clin Endocrinol Metab 94: 410-415.

3. DeFronzo RA, Bonadonna RC, Ferrannini E (1992) Pathogenesis of NIDDM. A balanced overview. Diabetes Care 15: 318-368.

4. Bergman RN, Ider YZ, Bowden CR, Cobelli C (1979) Quantitative estimation of insulin sensitivity. Am J Physiol 236: E667-677.

5. Lorenzo C, Wagenknecht LE, Rewers MJ, Karter AJ, Bergman RN, et al. (2010) Disposition index, glucose effectiveness, and conversion to type 2 diabetes: the Insulin Resistance Atherosclerosis Study (IRAS). Diabetes Care 33: 2098-2103.

6. Hanley AJ, Wagenknecht LE, D'Agostino RB, Jr., Zinman B, Haffner SM (2003) Identification of sub- jects with insulin resistance and beta-cell dysfunction using alternative definitions of the metabolic syndrome. Diabetes 52: 2740-2747.

7. Cheal KL, Abbasi F, Lamendola C, McLaughlin T, Reaven GM, et al. (2004) Relationship to insulin resistance of the adult treatment panel III diagnostic criteria for identification of the metabolic syndrome. Diabetes 53: $1195-1200$.

8. Laakso M (1993) How good a marker is insulin level for insulin resistance? Am J Epidemiol 137: 959-965.

9. Howard BV, Mayer-Davis EJ, Goff D, Zaccaro DJ, Laws A, et al. (1998) Relationships between insulin resistance and lipoproteins in nondiabetic African Americans, Hispanics, and non-Hispanic whites: the Insulin Resistance Atherosclerosis Study. Metabolism 47: 1174-1179.

10. Gower BA, Ard JD, Hunter GR, Fernandez J, Ovalle F (2007) Elements of the metabolic syndrome: association with insulin sensitivity and effects of ethnicity. Metab Syndr Relat Disord 5: 77-86.

11. American Diabetes Association (2012) Diagnosis and Classfication of Diabetes Mellitus. Diabetes Care 35 Suppl 1: S64-71. 
12. McAuley KA, Williams SM, Mann JI, Walker RJ, Lewis-Barned NJ, et al. (2001) Diagnosing insulin resistance in the general population. Diabetes Care 24: 460-464.

13. Danadian K, Balasekaran G, Lewy V, Meza MP, Robertson R, et al. (1999) Insulin sensitivity in AfricanAmerican children with and without family history of type 2 diabetes. Diabetes Care 22: 1325-1329.

14. Chiu KC, Chuang LM, Yoon C (2001) Comparison of measured and estimated indices of insulin sensitivity and beta cell function: impact of ethnicity on insulin sensitivity and beta cell function in glucose-tolerant and normotensive subjects. J Clin Endocrinol Metab 86: $1620-1625$.

15. Alford FP, Henriksen JE, Rantzau C, Vaag A, Hew LF, et al. (1998) Impact of family history of diabetes on the assessment of beta-cell function. Metabolism 47: 522528.

16. Katz A, Nambi SS, Mather K, Baron AD, Follmann DA, et al. (2000) Quantitative insulin sensitivity check index: a simple, accurate method for assessing insulin sensitivity in humans. J Clin Endocrinol Metab 85: 2402-2410.

17. Stumvoll M, Mitrakou A, Pimenta W, Jenssen T, YkiJarvinen H, et al. (2000) Use of the oral glucose tolerance test to assess insulin release and insulin sensitivity. Diabetes Care 23: 295-301.

18. van Haeften TW, Pimenta W, Mitrakou A, Korytkowski M, Jenssen T, et al. (2002) Disturbances in beta-cell function in impaired fasting glycemia. Diabetes 51 Suppl 1: S265-270.

19. van Haeften TW, Dubbeldam S, Zonderland ML, Erkelens DW (1998) Insulin secretion in normal glucose-tolerant relatives of type 2 diabetic subjects. Assessments using hyperglycemic glucose clamps and oral glucose tolerance tests. Diabetes Care 21: 278-282.

20. Matthews DR, Hosker JP, Rudenski AS, Naylor BA, Treacher DF, et al. (1985) Homeostasis model assessment: insulin resistance and beta-cell function from fasting plasma glucose and insulin concentrations in man. Diabetologia 28: 412-419.

21. Haffner SM, Miettinen H, Gaskill SP, Stern MP (1995) Decreased insulin secretion and increased insulin resistance are independently related to the 7-year risk of NIDDM in Mexican-Americans. Diabetes 44: 13861391.

22. Phillips DI, Clark PM, Hales CN, Osmond C (1994) Understanding oral glucose tolerance: comparison of glucose or insulin measurements during the oral glu- cose tolerance test with specific measurements of insulin resistance and insulin secretion. Diabet Med 11: 286292.

23. Kloppel G, Lohr M, Habich K, Oberholzer M, Heitz PU (1985) Islet pathology and the pathogenesis of type 1 and type 2 diabetes mellitus revisited. Surv Synth Pathol Res 4: 110-125.

24. Torrens JI, Skurnick J, Davidow AL, Korenman SG, Santoro N, et al. (2004) Ethnic differences in insulin sensitivity and beta-cell function in premenopausal or early perimenopausal women without diabetes: the Study of Women's Health Across the Nation (SWAN). Diabetes Care 27: 354-361.

25. Bardini G, Dicembrini I, Rotella CM, Giannini S (2011) Correlation between HDL cholesterol levels and betacell function in subjects with various degree of glucose tolerance. Acta Diabetol 50: 277-281.

26. Barter PJ (2011) The causes and consequences of low levels of high density lipoproteins in patients with diabetes. Diabetes Metab J 35: 101-106.

27. von Eckardstein A, Sibler RA (2011) Possible contributions of lipoproteins and cholesterol to the pathogenesis of diabetes mellitus type 2. Curr Opin Lipidol 22: 26-32.

28. Soro-Paavonen A, Naukkarinen J, Lee-Rueckert M, Watanabe H, Rantala E, et al. (2007) Common ABCA1 variants, HDL levels, and cellular cholesterol efflux in subjects with familial low HDL. J Lipid Res 48: 14091416.

29. Vergeer M, Brunham LR, Koetsveld J, Kruit JK, Verchere CB, et al. (2010) Carriers of loss-of-function mutations in ABCA1 display pancreatic beta-cell dysfunction. Diabetes Care 33: 869-874.

30. Oda E, Kawai R (2011) Changes in body weight are significantly associated with changes in fasting plasma glucose and HDL cholesterol in Japanese men without abdominal obesity (waist circumference $<85 \mathrm{~cm}$ ). Acta Diabetol 48: 143-148.

31. Haffner SM, D’Agostino R, Jr., Mykkanen L, Tracy R, Howard B, et al. (1999) Insulin sensitivity in subjects with type 2 diabetes. Relationship to cardiovascular risk factors: the Insulin Resistance Atherosclerosis Study. Diabetes Care 22: 562-568.

32. Rasouli N, Hale T, Kahn SE, Spencer HJ, Elbein SC (2005) Effects of short-term experimental insulin resistance and family history of diabetes on pancreatic beta-cell function in nondiabetic individuals. $J$ Clin Endocrinol Metab 90: 5825-5833. 